\title{
Pseudomonas grimontii sp. nov.
}

Laboratoire de

Bactériologie, Faculté de

Pharmacie, 3 Rue du Pr. Laguesse, BP 83, 59006 Lille Cedex, France

\author{
Nader Baïda, Asmae Yazourh, Elisabeth Singer and Daniel Izard
}

Author for correspondence: Daniel Izard. Tel: +3303209640 37. Fax: +330320397244. e-mail: daniel.izard@wanadoo.fr

The vernacular name 'fluorescent Pseudomonas group 97-514' was coined for a group of $\mathbf{4 3}$ strains isolated from two French natural mineral waters. All these strains were Gram-negative, rod-shaped and motile by means of a single polar flagellum. They produced fluorescent pigment (pyoverdin) on King B medium, catalase and cytochrome oxidase. They were capable of respiratory but not fermentative metabolism. They were not able to accumulate poly- $\beta$ hydroxybutyrate and possessed an arginine dihydrolase system. DNA-DNA relatedness studies (S1 nuclease method) showed that the 43 strains of 'fluorescent Pseudomonas group 97-514' formed a genetically homogeneous group (DNA-DNA relatedness ranged from 70 to $100 \%$ ). A total of 76 strains representing well-known or partially characterized species of the genus Pseudomonas sensu stricto had 7-56\% DNA hybridization with strain CFML 97$5^{1}{ }^{\top}$. The highest DNA binding values were found with Pseudomonas veronii CIP $104663^{\top}$ (52\%), Pseudomonas rhodesiae CIP 104664' (56\%), Pseudomonas marginalis ATCC $10844^{\top}$ (56\%), Pseudomonas gessardii CIP $105469^{\top}(53 \%)$ and Pseudomonas cedrella CIP $105541^{\top}(52 \%)$. Their unrelatedness was confirmed by $\Delta T_{m}$ values greater than $7{ }^{\circ} \mathrm{C}$. On the basis of the results of phenotypic and DNA-DNA hybridization studies, a novel Pseudomonas species, Pseudomonas grimontii sp. nov., is proposed for the $\mathbf{4 3}$ strains of ' fluorescent Pseudomonas group 97-514'. The type strain is strain CFML 97-514 ${ }^{\top}$ ( $=$ CIP 106645' $=$ ATCC BAA-140'). The $\mathrm{G}+\mathrm{C}$ content of the DNA of the type strain was $58 \mathrm{~mol} \%$. A comparison of the complete 16S rRNA gene sequence of the type strain CFML 97-514 ${ }^{\top}$ and the sequence of other strains of the genus Pseudomonas revealed that the novel species fell within the 'Pseudomonas fluorescens intrageneric cluster'. Members of $P$. grimontii grew at $4{ }^{\circ} \mathrm{C}$ but not at $41{ }^{\circ} \mathrm{C}$. They were able to use $D$-xylose, $\alpha$-L-rhamnose, $\alpha$-aminobutyrate, meso-erythritol and itaconate as sole sources of carbon and energy and formed levan from sucrose. Strains do not possess lecithinase or Tween esterase activities. The clinical significance of $P$. grimontii is unknown.

Keywords: Pseudomonas grimontii sp. nov., polyphasic taxonomy, mineral waters

\section{INTRODUCTION}

In recent years, Pseudomonas strains have been studied with increasing interest because of their importance in medical, food and environmental microbiology and phytopathology. The purpose of our research group is the taxonomic study of 'fluorescent' Pseudomonas strains isolated from natural mineral waters. In recent years, we have described seven novel species isolated

The EMBL accession number for the $16 \mathrm{~S}$ rRNA gene sequence of $P$. grimontii CFML $97-514^{\top}$ is AF268029. from natural mineral waters: Pseudomonas rhodesiae (Coroler et al., 1996), Pseudomonas veronii (Elomari et al., 1996), Pseudomonas jessenii and Pseudomonas mandelii (Verhille et al., 1999a), Pseudomonas gessardii and Pseudomonas migulae (Verhille et al., 1999b) and Pseudomonas brenneri (Baïda et al., 2001).

The vernacular name "fluorescent Pseudomonas group 97-514' was coined for 43 strains isolated from two natural mineral waters and not identified as any of our previously described species. All strains were able to hydrolyse starch, which is an unusual property among fluorescent Pseudomonas (Palleroni, 1984), except 


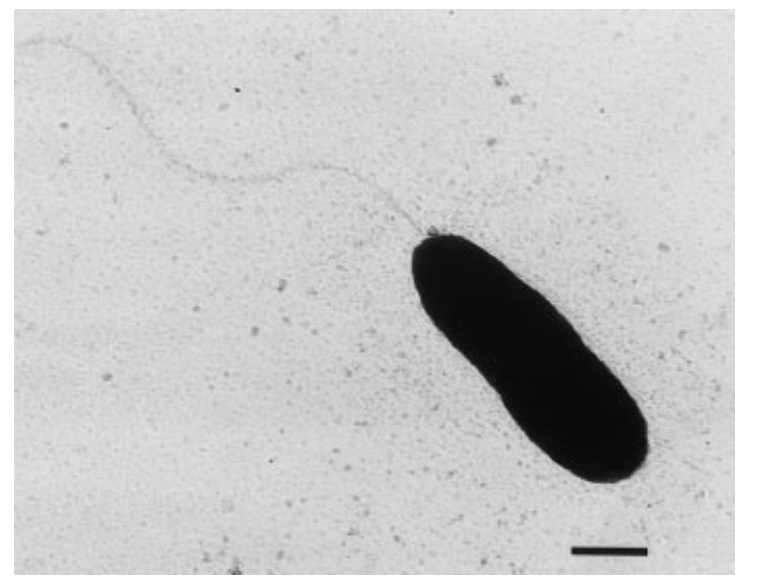

Fig. 1. Electron micrograph of a cell of $P$. grimontii sp. nov. CFML $97-514^{\top}$ showing the single polar flagellum. Bar, $0.5 \mu \mathrm{m}$. the recently described species $P$. brenneri (Baïda et al., 2001). The outcome of this polyphasic taxonomic study is the description of a novel species, Pseudomonas grimontii sp. nov.

\section{METHODS}

Bacterial strains. The 119 bacterial strains used in this study (Tables 2 and 3 ) included 43 wild strains isolated from two French natural mineral waters after bottling and identified as 'fluorescent Pseudomonas' and 76 type or collection strains from official collections, which were used for control purposes; they were chosen as representatives of authentic Pseudomonas species. All bacteria were cultured on MuellerHinton medium at $30^{\circ} \mathrm{C}$.

Flagellation study. Negative staining was done with fixed cells of the type strain of $P$. grimontii sp. nov., CFML 97$514^{\mathrm{T}}$, according to the method described by Hoeniger (1965).

Table 1. Characteristics that differentiate $P$. grimontii sp. nov. from various members of Pseudomonas sensu stricto

Taxa: 1, P. grimontii sp. nov.; 2, Pseudomonas aeruginosa; 3, P. fluorescens biovar I; 4, P. fluorescens biovar II; 5, P. fluorescens biovar III; 6, P. fluorescens biovar IV; 7, P. fluorescens biovar V; 8, Pseudomonas chlororaphis; 9 , Pseudomonas putida biovar A; 10, P. putida biovar B; 11, P. veronii; 12, P. rhodesiae; 13, P. gessardii; 14, P. migulae; 15, P. mandelii; 16, P. jessenii; 17, P. brenneri; 18, Pseudomonas libanensis; 19, P. cedrella; 20, P. orientalis. Characteristics are scored as:,$- \geqslant 90 \%$ of strains negative;,$+ \geqslant 90 \%$ of strains positive; $\mathrm{d}, 11-89 \%$ of strains positive; ND, not determined. Data were taken from Palleroni (1984), Elomari et al. (1996), Coroler et al. (1996), Verhille et al. (1999a, b), Dabboussi et al. (1999a, b) and Baïda et al. (2001).

\begin{tabular}{|c|c|c|c|c|c|c|c|c|c|c|c|c|c|c|c|c|c|c|c|c|}
\hline Characteristic & 1 & 2 & 3 & 4 & 5 & 6 & 7 & 8 & 9 & 10 & 11 & 12 & 13 & 14 & 15 & 16 & 17 & 18 & 19 & 20 \\
\hline Pyocyanin production & - & + & - & - & - & - & - & - & - & - & - & - & - & - & - & - & - & - & - & - \\
\hline Denitrification & + & + & - & + & + & + & - & + & - & - & + & - & $\mathrm{d}$ & + & + & - & + & - & $\mathrm{d}$ & - \\
\hline Growth at $4{ }^{\circ} \mathrm{C}$ & + & - & + & + & + & + & $\mathrm{d}$ & + & $\mathrm{d}$ & + & + & + & + & + & + & + & + & + & + & + \\
\hline Growth at $41{ }^{\circ} \mathrm{C}$ & - & + & - & - & - & - & - & - & - & - & - & - & - & - & - & - & - & - & - & - \\
\hline Lecithinase & - & - & + & $\mathrm{d}$ & + & + & $\mathrm{d}$ & + & - & - & - & + & $\mathrm{d}$ & + & - & - & + & + & - & $\mathrm{d}$ \\
\hline Starch hydrolysis & + & - & - & - & - & - & - & - & - & - & - & - & - & - & - & - & + & - & - & - \\
\hline Levan & + & - & + & + & - & + & - & + & - & - & + & + & + & + & + & + & + & + & + & + \\
\hline \multicolumn{21}{|l|}{ Assimilation of: } \\
\hline D-Ribose & + & + & + & + & $\mathrm{d}$ & + & $\mathrm{d}$ & + & $\mathrm{d}$ & $\mathrm{d}$ & + & + & + & + & + & + & + & + & + & + \\
\hline D-Xylose & + & - & + & $\mathrm{d}$ & $\mathrm{d}$ & $\mathrm{d}$ & $\mathrm{d}$ & - & $\mathrm{d}$ & $\mathrm{d}$ & + & + & - & + & + & $\mathrm{d}$ & - & + & + & + \\
\hline$\alpha$-L-Rhamnose & + & - & - & $\mathrm{d}$ & $\mathrm{d}$ & - & $\mathrm{d}$ & - & - & - & - & - & - & - & $\mathrm{d}$ & $\mathrm{d}$ & + & $\mathrm{d}$ & $\mathrm{d}$ & $\mathrm{d}$ \\
\hline D-Mannose & + & - & + & + & + & + & $\mathrm{d}$ & + & $\mathrm{d}$ & $\mathrm{d}$ & + & + & + & + & + & + & + & + & + & + \\
\hline D-Mannitol & + & + & + & + & $\mathrm{d}$ & + & $\mathrm{d}$ & + & $\mathrm{d}$ & $\mathrm{d}$ & + & + & + & + & + & + & + & + & + & + \\
\hline D-Trehalose & + & - & + & + & $\mathrm{d}$ & + & $\mathrm{d}$ & + & - & - & $\mathrm{d}$ & + & + & + & + & $\mathrm{d}$ & + & $\mathrm{d}$ & + & + \\
\hline 2-Keto-D-gluconate & + & + & + & + & + & $\mathrm{d}$ & + & + & $\mathrm{d}$ & + & + & + & + & + & $\mathrm{d}$ & + & + & + & + & + \\
\hline Mucate & + & - & + & + & $\mathrm{d}$ & + & + & + & $\mathrm{d}$ & + & $\mathrm{d}$ & + & $\mathrm{d}$ & + & + & + & + & + & + & + \\
\hline Malonate & + & + & + & + & $\mathrm{d}$ & + & $\mathrm{d}$ & + & $\mathrm{d}$ & + & + & + & + & + & + & + & + & + & + & + \\
\hline D-Tartrate & $\mathrm{d}$ & - & - & $\mathrm{d}$ & - & - & $\mathrm{d}$ & - & $\mathrm{d}$ & $\mathrm{d}$ & - & - & - & - & - & - & - & - & $\mathrm{d}$ & - \\
\hline meso-Tartrate & - & - & - & - & $\mathrm{d}$ & - & $\mathrm{d}$ & - & $\mathrm{d}$ & - & - & - & - & + & + & + & - & $\mathrm{d}$ & - & - \\
\hline$m$-Hydroxybenzoate & $\mathrm{d}$ & - & - & - & - & - & - & $\mathrm{d}$ & $\mathrm{d}$ & $\mathrm{d}$ & - & - & $\mathrm{d}$ & - & $\mathrm{d}$ & $\mathrm{d}$ & - & - & - & - \\
\hline$\alpha$-Aminobutyrate & + & - & - & - & - & - & - & - & - & $\mathrm{d}$ & + & + & + & + & + & + & + & + & + & + \\
\hline meso-Erythritol & + & - & $\mathrm{d}$ & $\mathrm{d}$ & + & - & $\mathrm{d}$ & - & - & - & + & - & + & - & - & - & + & + & + & - \\
\hline D-Sorbitol & + & - & + & + & $\mathrm{d}$ & + & $\mathrm{d}$ & - & - & $\mathrm{d}$ & + & + & - & - & + & $\mathrm{d}$ & - & + & + & + \\
\hline myo-Inositol & + & - & $\mathrm{d}$ & + & $\mathrm{d}$ & + & $\mathrm{d}$ & + & - & - & + & + & + & - & + & $\mathrm{d}$ & + & + & + & + \\
\hline Adonitol & - & - & + & - & $\mathrm{d}$ & - & $\mathrm{d}$ & - & - & - & - & - & + & - & $\mathrm{d}$ & $\mathrm{d}$ & + & + & + & + \\
\hline Benzoate & $\mathrm{d}$ & + & $\mathrm{d}$ & $\mathrm{d}$ & $\mathrm{d}$ & + & $\mathrm{d}$ & + & $\mathrm{d}$ & + & + & - & $\mathrm{d}$ & + & + & + & + & - & - & - \\
\hline Itaconate & + & + & + & $\mathrm{d}$ & $\mathrm{d}$ & - & $\mathrm{d}$ & + & $\mathrm{d}$ & - & ND & ND & $\mathrm{d}$ & - & $\mathrm{d}$ & $\mathrm{d}$ & + & + & - & $\mathrm{d}$ \\
\hline L-Histidine & - & + & + & $\mathrm{d}$ & + & + & $\mathrm{d}$ & + & + & + & $\mathrm{d}$ & + & - & - & - & - & - & - & - & - \\
\hline L-Tryptophan & - & $\mathrm{d}$ & + & $\mathrm{d}$ & $\mathrm{d}$ & - & $\mathrm{d}$ & + & - & + & + & - & - & - & - & - & - & - & - & - \\
\hline Histamine & - & + & $\mathrm{d}$ & - & $\mathrm{d}$ & - & $\mathrm{d}$ & $\mathrm{d}$ & $\mathrm{d}$ & + & - & - & - & + & + & + & - & - & - & - \\
\hline Tryptamine & - & - & - & $\mathrm{d}$ & $\mathrm{d}$ & - & - & - & $\mathrm{d}$ & + & - & - & + & - & - & - & $\mathrm{d}$ & - & - & - \\
\hline Trigonelline & + & - & $\mathrm{d}$ & $\mathrm{d}$ & $\mathrm{d}$ & - & $\mathrm{d}$ & - & $\mathrm{d}$ & + & + & - & + & - & - & + & - & - & + & + \\
\hline
\end{tabular}


The stained bacteria were examined with a JEOL type 100 CX transmission electron microscope.

Phenotypic characterization. Forty-two conventional tests were performed as utilized by Gavini et al. (1989). Carbon source utilization tests were studied using the Biotype-100 system (bioMérieux) as described by Grimont et al. (1996). Enzymic tests were performed on API ZYM strips (bioMérieux). The enzymic activities tested included four esterase, seven peptidase and eight oxidase activities. These enzymic activities were studied at $30^{\circ} \mathrm{C}$ for $4 \mathrm{~h}$. The tests were scored according to the manufacturer's recommendations.

DNA extraction. Strains were grown on Mueller-Hinton medium plates. Chromosomal DNA was extracted as described previously (Marmur, 1961; Beji et al., 1987). The purity and quality of each DNA preparation were checked by determining the $A_{260} / A_{280}$ and $A_{260} / A_{230}$ ratios, as described by Marmur \& Doty (1962).

DNA-DNA hybridization. Native DNA was labelled in vitro by nick translation with tritium-labelled nucleotides (Grimont et al., 1980). The S1 nuclease/trichloroacetic acid method for hybridization has been described previously (Crosa et al., 1973; Grimont et al., 1980). Each hybridization experiment was at least done twice.

Thermal stability of duplexes. The thermal stability of duplexes was determined by using the method of Crosa et al. (1973). The divergence between DNAs was estimated by determining the $\Delta T_{\mathrm{m}}$ value.

DNA base composition. $\mathrm{G}+\mathrm{C}$ content was calculated from the thermal denaturation curve by using the equation of De Ley (1970).

16S rDNA sequence determination. The almost complete $16 \mathrm{~S}$ rRNA gene sequence was determined for strain CFML 97$514^{\mathrm{T}}$ by direct PCR sequencing. DNA was amplified by using two 16S rRNA universal primers, $\mathrm{pA}$ and $\mathrm{pH}$, described previously (Edwards et al., 1989). PCR amplification were performed as described by Baïda et al. (2001).

Phylogenetic analysis. Using the program CLUSTAL $\mathrm{X}$, which is a major up-date of the multiple alignment program CLUSTAL W (Thompson et al., 1994), the primary structure was aligned and compared with the primary structures of reference strains of the genus Pseudomonas sensu stricto (Kersters et al., 1996) obtained from the EMBL sequence database. Nucleotide substitution rates ( $K_{\text {nuc }}$ values) were calculated (Kimura, 1980) and a phylogenetic tree was constructed by the neighbour-joining method of Saitou \& Nei (1987). The tree topology was tested by a bootstrap analysis of 1000 resamplings (Felsenstein, 1985).

Nucleotide sequence accession numbers. The strain designations and accession numbers of 16S rRNA gene sequences of other species that were compared with the CFML 97-514 ${ }^{\mathrm{T}}$ sequence in this study are displayed in Fig. 2.

\section{RESULTS AND DISCUSSION}

Single polar flagella (Fig. 1) were observed by scanning electron microscopy on cells of Pseudomonas grimontii CFML $97-514^{\mathrm{T}}$. A summary of phenotypic properties is shown in Table 1 and in the description of the novel species.

Hybridization experiments performed with the labelled DNA of $P$. grimontii CFML $97-514^{\mathrm{T}}$ and the 42
Table 2. DNA-DNA hybridization values between $P$. grimontii CFML $97-514^{\top}$ and other mineral water isolates

Labelled DNA from strain CFML 97-514 ${ }^{\mathrm{T}}$ was used. All strains were isolated from two French natural mineral waters (A and B) after bottling. CFML, Collection de la Faculté de Médecine, Lille, France; RBR, relative binding ratio.

\begin{tabular}{|c|c|c|}
\hline Strain & $\begin{array}{c}\text { Origin of } \\
\text { mineral water }\end{array}$ & $\begin{array}{c}\operatorname{RBR}(\%) \\
{\left[\Delta T_{m}\left({ }^{\circ} \mathrm{C}\right)\right]}\end{array}$ \\
\hline CFML 97-514 & $\mathrm{B}$ & $100[0]$ \\
\hline CFML 97-492 & $\mathrm{B}$ & 99 \\
\hline CFML 97-498 & $\mathrm{B}$ & 99 \\
\hline CFML 97-505 & B & 97 \\
\hline CFML 97-528 & B & 96 \\
\hline CFML 97-521 & $\mathrm{B}$ & 96 \\
\hline CFML 97-520 & B & 96 \\
\hline CFML 97-525 & $\mathrm{B}$ & 96 \\
\hline CFML 97-493 & $\mathrm{B}$ & 96 \\
\hline CFML 97-509 & $\mathrm{B}$ & 96 \\
\hline CFML 97-485 & B & 95 \\
\hline CFML 97-501 & $\mathrm{B}$ & 95 \\
\hline CFML 97-508 & B & 95 \\
\hline CFML 97-510 & $\mathrm{B}$ & 95 \\
\hline CFML 97-523 & B & 95 \\
\hline CFML 97-490 & $\mathrm{B}$ & 94 \\
\hline CFML 97-483 & $\mathrm{B}$ & 94 \\
\hline CFML 97-530 & B & 94 \\
\hline CFML 97-484 & $\mathrm{B}$ & 94 \\
\hline CFML 97-491 & $\mathrm{B}$ & 93 \\
\hline CFML 97-486 & $\mathrm{B}$ & 92 \\
\hline CFML 97-515 & $\mathrm{B}$ & 91 \\
\hline CFML 97-517 & $\mathrm{B}$ & 91 \\
\hline CFML 97-489 & $\mathrm{B}$ & 88 \\
\hline CFML 97-507 & $\mathrm{B}$ & 87 \\
\hline CFML 97-512 & $\mathrm{B}$ & 87 \\
\hline CFML 97-519 & $\mathrm{B}$ & 87 \\
\hline CFML 97-467 & A & 86 \\
\hline CFML 96-331 & A & 85 \\
\hline CFML 97-506 & $\mathrm{B}$ & 85 \\
\hline CFML 97-504 & $\mathrm{B}$ & 84 \\
\hline CFML 97-494 & B & 84 \\
\hline CFML 97-503 & B & 83 \\
\hline CFML 97-592 & B & 82 \\
\hline CFML 97-495 & $\mathrm{B}$ & 81 \\
\hline CFML 97-502 & $\mathrm{B}$ & 79 \\
\hline CFML 97-481 & B & 78 \\
\hline CFML 97-524 & $\mathrm{B}$ & 78 \\
\hline CFML 96-323 & A & $75[0]$ \\
\hline CFML 96-347 & A & $75[0]$ \\
\hline CFML 96-336 & A & $75[0]$ \\
\hline CFML 97-500 & $\mathrm{B}$ & $73[0]$ \\
\hline CFML 97-516 & $\mathrm{B}$ & $70[1]$ \\
\hline
\end{tabular}

other isolates of 'fluorescent Pseudomonas group 97514 ' showed that strains were $70-100 \%$ related to CFML $97-514^{\mathrm{T}}$ (Table 2). DNA-DNA hybridization values between strain CFML 97-514 ${ }^{\mathrm{T}}$ and other previously described species of the genus Pseudomonas 
Table 3. Levels of DNA relatedness of $P$. grimontii CFML $97-514^{\top}$ to different type and collection strains of the genus Pseudomonas

Labelled DNA from strain CFML 97-514 ${ }^{\mathrm{T}}$ was used. ATCC, American Type Culture Collection, Manassas, VA, USA; CCEB, Culture Collection of Entomogenous Bacteria, Institute of Entomology, Czechoslovak Academy of Sciences, Prague, Czech Republic; CCM, Czechoslovak Collection of Microorganisms, University of Brno, Czech Republic; CCUG, Culture Collection, University of Göteborg, Göteborg, Sweden; CIP, Collection de l'Institut Pasteur, Paris, France; DSM, Deutsche Sammlung von Mikroorganismen und Zellkulturen, Braunschweig, Germany; NCPPB, National Collection of Plant-pathogenic bacteria, Harpenden, UK; CFBP, Collection Française des Bactéries Phytopathogènes, Angers, France.

\begin{tabular}{|c|c|c|c|}
\hline Source of unlabelled DNA & $\begin{array}{c}\operatorname{RBR}(\%) \\
{\left[\Delta T_{\mathrm{m}}\left({ }^{\circ} \mathrm{C}\right)\right]}\end{array}$ & Source of unlabelled DNA & $\begin{array}{c}\operatorname{RBR}(\%) \\
{\left[\Delta T_{\mathrm{m}}\left({ }^{\circ} \mathrm{C}\right)\right]}\end{array}$ \\
\hline P. aeruginosa & & P. savastanoi (cont.) & \\
\hline ATCC $10145^{\mathrm{T}}$ & 20 & CFBP 1838 & 10 \\
\hline ATCC 27853 & 11 & Pseudomonas viridiflava ATCC $13223^{\mathrm{T}}$ & 10 \\
\hline ATCC 15692 & 38 & Pseudomonas syringae ATCC $19310^{\mathrm{T}}$ & 15 \\
\hline P. fluorescens biovar I & & Pseudomonas cichorii DSM 50259 & 17 \\
\hline ATCC $13525^{\mathrm{T}}$ & 49 & Pseudomonas agarici ATCC $25941^{\mathrm{T}}$ & 20 \\
\hline ATCC 17563 & 40 & Pseudomonas asplenii ATCC $23835^{\mathrm{T}}$ & 23 \\
\hline P. fluorescens biovar II & & Pseudomonas caricapapayae NCPPB $1873^{\mathrm{T}}$ & 19 \\
\hline CFBP 12301 & 16 & Pseudomonas tolaasii & \\
\hline ATCC 17482 & 33 & NCPPB $2192^{\mathrm{T}}$ & 46 \\
\hline CFBP 11346 & 33 & NCPPB 1616 & 49 \\
\hline CFBP 12296 & 40 & Pseudomonas stutzeri & \\
\hline CFBP 11759 & 15 & ATCC $17588^{\mathrm{T}}$ & 10 \\
\hline CFBP 11776 & 16 & ATCC 17587 & 11 \\
\hline P. fluorescens biovar III & & ATCC 17591 & 28 \\
\hline ATCC 17559 & 19 & ATCC 17686 & 39 \\
\hline ATCC 17571 & 36 & Pseudomonas mendocina & \\
\hline ATCC 17400 & 36 & ATCC $25411^{\mathrm{T}}$ & 13 \\
\hline P. fluorescens biovar IV & & ATCC 25412 & 10 \\
\hline DSM 50415 & 35 & Pseudomonas alcaligenes ATCC $14909^{\mathrm{T}}$ & 10 \\
\hline ATCC 12983 & 31 & Pseudomonas pseudoalcaligenes & \\
\hline P. fluorescens biovar $\mathrm{V}$ & & ATCC $17440^{\mathrm{T}}$ & 16 \\
\hline ATCC 15916 & 46 & ATCC 12815 & 18 \\
\hline ATCC 17518 & 48 & Pseudomonas fragi & \\
\hline ATCC 17573 & 40 & ATCC $4973^{\mathrm{T}}$ & 39 \\
\hline DSM 50148 & 27 & ATCC 27362 & 16 \\
\hline ATCC 17386 & 25 & Pseudomonas mucidolens CIP $103298^{\mathrm{T}}$ & 38 \\
\hline P. putida biovar A & & Pseudomonas ficuserectae CFBP $3224^{\mathrm{T}}$ & 7 \\
\hline ATCC $12633^{\mathrm{T}}$ & 38 & Pseudomonas synxantha CIP 5922 & 36 \\
\hline DSM 50208 & 21 & 'Pseudomonas coronafaciens' CFBP 2216 & 10 \\
\hline P. putida biovar B & & Pseudomonas corrugata CIP $105514^{\mathrm{T}}$ & 27 \\
\hline ATCC 17484 & 25 & Pseudomonas cannabina CFBP $2341^{\mathrm{T}}$ & 18 \\
\hline ATCC 17430 & 24 & P. chlororaphis & \\
\hline CCUG 1317 & 20 & DSM $50083^{T}$ & 39 \\
\hline P. veronii CIP $104663^{\mathrm{T}}$ & $52[10]$ & ATCC 9447 & 45 \\
\hline P. rhodesiae CIP $104664^{\mathrm{T}}$ & $56[10]$ & ATCC 17414 & 30 \\
\hline Pseudomonas monteilii CIP $104883^{\mathrm{T}}$ & 35 & Pseudomonas aureofaciens & \\
\hline P. jessenii CIP $105274^{\mathrm{T}}$ & 18 & CCEB $518^{\mathrm{T}}$ & 26 \\
\hline P. mandelii CIP $105273^{\mathrm{T}}$ & 29 & ATCC 17415 & 31 \\
\hline P. gessardii CIP $105469^{\mathrm{T}}$ & $53[11]$ & Pseudomonas lundensis & \\
\hline P. migulae CIP $105470^{\mathrm{T}}$ & 32 & CCM $573^{\mathrm{T}}$ & 19 \\
\hline P. libanensis CIP $105460^{\mathrm{T}}$ & 37 & CCUG 18758 & 14 \\
\hline P. orientalis CIP $105540^{\mathrm{T}}$ & 36 & Pseudomonas flavescens & \\
\hline P. cedrella CIP $105541^{\mathrm{T}}$ & $52[9]$ & CIP $104204^{\mathrm{T}}$ & 38 \\
\hline Pseudomonas fuscovaginae NCPPB $3085^{\mathrm{T}}$ & 16 & CIP 104205 & 13 \\
\hline Pseudomonas savastanoi & & P. marginalis ATCC $10844^{\mathrm{T}}$ & $56[7]$ \\
\hline CFBP $1670^{\mathrm{T}}$ & 15 & P. brenneri CIP $106646^{\mathrm{T}}$ & 33 \\
\hline CFBP 2088 & 20 & & \\
\hline
\end{tabular}




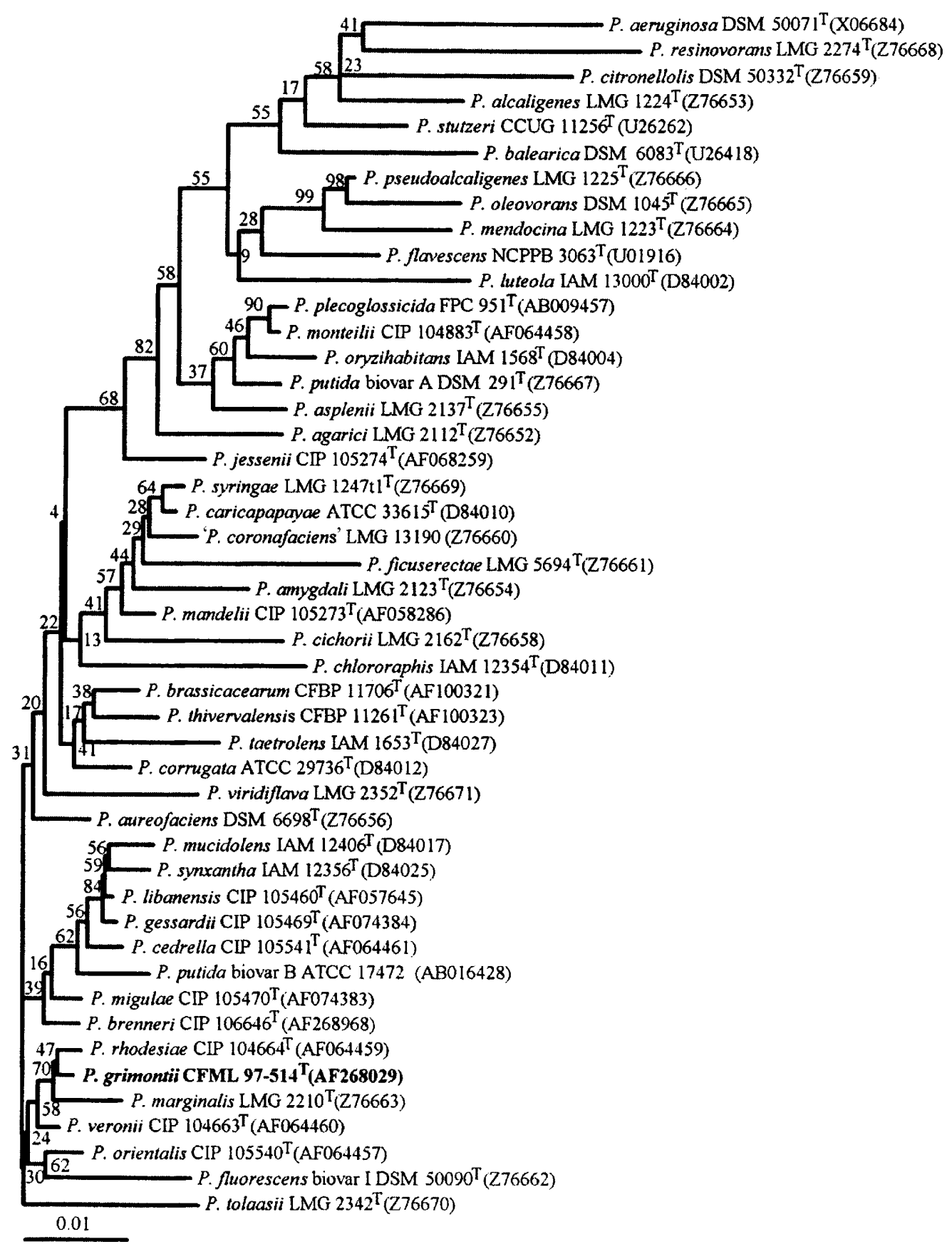

Fig. 2. Unrooted tree, constructed by the neighbour-joining method, showing the phylogenetic relationships of $P$. grimontii sp. nov. CFML $97-514^{\top}$ and other species of the genus Pseudomonas sensu stricto. Numbers at nodes show the level of bootstrap support based on data for 1000 replications. Bar, 0.01 substitutions per nucleotide position.

varied from 7 to $56 \%$, and their unrelatedness was confirmed by $\Delta T_{\mathrm{m}}$ values greater than $7^{\circ} \mathrm{C}$ (Table 3). The DNA G $+\mathrm{C}$ content of Pseudomonas grimontii CFML $97-514^{\mathrm{T}}$ was $58 \mathrm{~mol} \%$.

The phylogenetic tree based on $16 \mathrm{~S}$ rDNA sequencing is displayed in Fig. 2. Percentage identities between strain CFML $97-514^{\mathrm{T}}$ and other Pseudomonas species ranged from 93 to $99 \%$. The highest similarity $(99 \%)$ was found with the five strains $P$. rhodesiae CIP
$104664^{\mathrm{T}}$, P. veronii CIP $104663^{\mathrm{T}}$, Pseudomonas orientalis CIP $105540^{\mathrm{T}}$, Pseudomonas marginalis LMG $2210^{\mathrm{T}}$ and $P$. brenneri CIP $106646^{\mathrm{T}}$, but they were clearly different in DNA-DNA hybridization (Table 3 ) and phenotypic characteristics (Table 1).

According to the our results, we conclude that members of 'fluorescent Pseudomonas group 97-514' belong to a novel species, for which we propose the name $P$. grimontii sp. nov. The novel species can be differ- 
entiated from other 'fluorescent' Pseudomonas species by several phenotypic features (Table 1).

\section{Description of Pseudomonas grimontii sp. nov.}

Pseudomonas grimontii (gri.mon'ti.i. N.L. gen. n. grimontii of Grimont, in honour of the French bacteriologist P. A. D. Grimont, for all his work in the field of bacterial taxonomy).

Cells are Gram-negative, asporogenous, rod-shaped and motile by means of a single polar flagellum (Fig. 1). Colonies are smooth on nutrient agar, circular, non-pigmented and haemolytic (98\% of strains) when grown on blood agar. All strains produce a fluorescent pigment on both King B medium and S1 medium (Gould et al., 1985). Arginine dihydrolase, tributyrin esterase ( $93 \%$ of strains), catalase and oxidase are also produced. Lysine and ornithine are not decarboxylated. Cells can reduce nitrate to nitrite and can grow at low salt concentrations $(0.8 \%)$ but not at high salt concentrations (5 or 7\%). Strains do not possess any DNase, coagulase, tetrathionate reductase $(95 \%$ of strains), elastase or Tween esterase activities. Fibrinolysis and acetyl-methylcarbinol (acetoin) tests are negative. Acid is formed from starch. Poly- $\beta$-hydroxybutyrate is not accumulated.

At least $90 \%$ of strains utilize the following substrates as carbon and energy sources: $\alpha$-D-glucose, $\beta$-Dfructose, D-galactose, L-arabinose, sucrose, D-arabitol, glycerol, D-glucosamine, D-saccharate, L-malate, cisaconitate, citrate, D-glucuronate, D-galacturonate, 5keto-D-gluconate, $\mathrm{N}$-acetyl D-glucosamine, D-gluconate, protocatechuate, $p$-hydroxybenzoate, quinate, betaine, putrescine, DL-lactate, caprate, caprylate, succinate, fumarate, glutarate, DL-glycerate, 3-hydroxybutyrate, L-aspartate, L-glutamate, L-proline, Dalanine, $\mathrm{L}$-alanine, $\mathrm{L}$-serine, propionate, $\mathrm{L}$-tyrosine and 2-oxoglutarate. The following compounds are not utilized by $90 \%$ or more of the strains : L-sorbose, $\alpha$-Dmelibiose, maltotriose, D-raffinose, maltose, $\alpha$-lactose, lactulose, 1-O-methyl $\beta$-galactopyranoside, 1-Omethyl $\alpha$-galactopyranoside, D-cellobiose, $\beta$-gentiobiose, 1- $O$-methyl $\beta$-D-glucopyranoside, palatinose, $\alpha$ L-fucose, D-melezitose, L-arabitol, xylitol, dulcitol, Dtagatose, maltitol, D-turanose, hydroxyquinoline $\beta$ glucuronide, 1- $O$-methyl $\alpha$-D-glucopyranoside, 3- $O$ methyl D-glucopyranose, L-tartrate, trans-aconitate, tricarballylate, phenylacetate, gentisate, 3-phenylpropionate and $m$-coumarate. The other characteristics are displayed in Table 1.

At least $90 \%$ of strains possess the following enzyme activities: esterase $\mathrm{C}_{4}$, alkaline phosphatase, esterase lipase $\mathrm{C}_{8}$ and leucine arylamidase. At least $90 \%$ of strains do not possess the following enzyme activities: trypsin, $\alpha$-chymotrypsin, $\alpha$-galactosidase, $\beta$-galactosidase, $\beta$-glucosidase, $N$-acetyl- $\beta$-glucosaminidase, $\beta$ glucuronidase, $\alpha$-glucosidase, $\alpha$-mannosidase and $\alpha$ fucosidase.

All strains have been isolated from natural mineral water. No clinical significance is known. The type strain is CFML $97-514^{\mathrm{T}}\left(=\mathrm{CIP} 106645^{\mathrm{T}}=\mathrm{ATCC}\right.$ BAA- $140^{\mathrm{T}}$ ).

\section{REFERENCES}

Baïda, N., Yazourh, A., Singer, E. \& Izard, D. (2001). Pseudomonas brenneri sp. nov., a new species isolated from natural mineral waters. Res Microbiol 152, 493-502.

Beji, A., Izard, D., Gavini, F., Leclerc, H., Leseine-Delstanche, M. \& Krembel, J. (1987). A rapid chemical procedure for isolation and purification of chromosomal DNA from Gram-negative bacilli. Anal Biochem 161, 18-23.

Coroler, L., Elomari, M., Hoste, B., Gillis, M., Izard, D. \& Leclerc, H. (1996). Pseudomonas rhodesiae sp. nov., a new species isolated from natural mineral waters. Syst Appl Microbiol 19, 600-607.

Crosa, J. H., Brenner, D. J. \& Falkow, S. (1973). Use of a single-strand specific nuclease for analysis of bacterial and plasmid deoxyribonucleic acid homo- and heteroduplex. J Bacteriol 115, 904-911.

Dabboussi, F., Hamze, M., Elomari, M., Verhille, S., Baïda, N., Izard, D. \& Leclerc, H. (1999a). Taxonomic study of bacteria isolated from Lebanese spring waters: proposal for Pseudomonas cedrella sp. nov. and Pseudomonas orientalis sp. nov. Res Microbiol 150, 303-316.

Dabboussi, F., Hamze, M., Elomari, M., Verhille, S., Baïda, N., Izard, D. \& Leclerc, H. (1999b). Pseudomonas libanensis sp. nov., a new species isolated from Lebanese spring waters. Int J Syst Bacteriol 49, 1091-1101.

De Ley, J. (1970). Reexamination of the association between melting point, buoyant density, and chemical base composition of deoxyribonucleic acid. $J$ Bacteriol 101, 738-754.

Edwards, U., Rogall, T., Blöcker, H., Emde, M. \& Böttger, E. C. (1989). Isolation and direct complete nucleotide determination of entire genes. Characterization of a gene coding for $16 \mathrm{~S}$ ribosomal RNA. Nucleic Acids Res 17, 7843-7853.

Elomari, M., Coroler, L., Hoste, B., Gillis, M., Izard, D. \& Leclerc, H. (1996). DNA relatedness among Pseudomonas strains isolated from natural mineral waters and proposal of Pseudomonas veronii sp. nov. Int J Syst Bacteriol 46, 1138-1144.

Felsenstein, J. (1985). Confidence limits on phylogenies: an approach using the bootstrap. Evolution 39, 783-791.

Gavini, F., Holmes, B., Izard, D., Beji, A., Bernigaud, A. \& Jakubczak, E. (1989). Numerical taxonomy of Pseudomonas alcaligenes, Pseudomonas pseudoalcaligenes, Pseudomonas mendocina, Pseudomonas stutzeri and related bacteria. Int J Syst Bacteriol 39, 135-144.

Gould, W. D., Hagedorn, C., Bardinelli, T. R. \& Zablotow, R. H. (1985). New selective media for enumeration and recovery of fluorescent pseudomonads from various habitats. Appl Environ Microbiol 49, 28-32.

Grimont, P. A. D., Popoff, M. Y., Grimont, F., Coynault, C. \& Lemelin, M. (1980). Reproducibility and correlation study of three deoxyribonucleic acid hybridization procedures. Curr Microbiol 4, 325-330.

Grimont, P. A. D., Vancanneyt, M., Lefèvre, M., Vandemeulebroecke, K., Vauterin, L., Brosch, R., Kersters, K. \& Grimont, F. (1996). Ability of Biolog and Biotype-100 systems to reveal the taxonomic diversity of the pseudomonads. Syst Appl Microbiol 19, 510-527.

Hoeniger, J. F. M. (1965). Development of flagella by Proteus mirabilis. J Gen Microbiol 40, 29-33.

Kersters, K., Ludwig, W., Vancanneyt, M., De Vos, P., Gillis, M. \& Schleifer, K. H. (1996). Recent changes in the classification of the pseudomonads: an overview. Syst Appl Microbiol 19, 465-477.

Kimura, M. (1980). A simple method for estimating evolutionary rates of base substitutions through comparative studies of nucleotide sequences. J Mol Evol 16, 111-120.

Marmur, J. (1961). A procedure for the isolation of deoxyribonucleic acid from microorganisms. J Mol Biol 3, 208-218.

Marmur, J. \& Doty, P. (1962). Determination of the base composition 
of deoxyribonucleic acid from its thermal denaturation temperature. $J$ Mol Biol 5, 109-118.

Palleroni, N. J. (1984). Genus I. Pseudomonas Migula 1894, $237^{\mathrm{AL}}$ (nom. cons. opin. 5, jud. comm. 1952, 237). In Bergey's Manual of Systematic Bacteriology, vol. 1, pp. 141-199. Edited by N. R. Krieg \& J. G. Holt. Baltimore: Williams \& Wilkins.

Saitou, N. \& Nei, M. (1987). The neighbor-joining method: a new method for reconstructing phylogenetic trees. Mol Biol Evol 4, 406-425.

Thompson, J. D., Higgins, D. G. \& Gibson, T. J. (1994). CLuSTAL w: improving the sensitivity of progressive multiple sequence alignment through sequence weighting, position-specific gap penalties and weight matrix choice. Nucleic Acids Res 22, 4673-4680.

Verhille, S., Baïda, N., Dabboussi, F., Izard, D. \& Leclerc, H. (1999a). Taxonomic study of bacteria isolated from natural mineral waters: proposal of Pseudomonas jessenii sp. nov. and Pseudomonas mandelii sp. nov. Syst Appl Microbiol 22, 45-58.

Verhille, S., Baïda, N., Dabboussi, F., Hamze, M., Izard, D. \& Leclerc, H. (1999b). Pseudomonas gessardii sp. nov. and Pseudomonas migulae sp. nov., two new species isolated from natural mineral waters. Int J Syst Bacteriol 49, 1559-1572. 